\title{
IN-HOME TELEREHABILITATION FOR POST-KNEE ARTHROPLASTY: A PILOT STUDY
}

\author{
MiCHEL TOUSIGNANT ${ }^{1}$, PATRICK BOISSY², HÉlÈnE CORRIVEAU ${ }^{1}$, \\ HÉLÈNE MOFFET ${ }^{3}$, FRANCOIS CABANA ${ }^{1}$ \\ ${ }^{1}$ FACULTY OF MEDICINE, UNIVERSITY OF SHERBROOKE, ${ }^{2}$ FACULTY OF PHYSICAL EDUCATION, \\ UNIVERSITY OF SHERBROOKE, ${ }^{3}$ FACULTY OF MEDICINE, LAVAL UNIVERSITY
}

\section{ABSTRACT}

The purpose of this study was to investigate the efficacy of in-home telerehabilitation as an alternative to conventional rehabilitation services following knee arthroplasty. Five community-living elders who had knee arthroplasty were recruited prior to discharge from an acute care hospital. A pre/post-test design without a control group was used for this pilot study. Telerehabilitation sessions (16) were conducted by two trained physiotherapists from a service center to the patient's home using H264 videoconference CODECs (Tandberg $550 \mathrm{MXP}$ ) connected at $512 \mathrm{~Kb}$ ls. Disability (range of motion, balance and lower body strength) and function (locomotor performance in walking and functional autonomy) were measured in face-to-face evaluations prior to and at the end of the treatments by a neutral evaluator. The satisfaction of the health care professional and patient was measured by questionnaire. Results are as follows. One participant was lost during follow-up. Clinical outcomes improved for all subjects and improvements were sustained two months post-discharge from in-home telerehabilitation. The satisfaction of the participants with in-home telerehabilitation services was very high. The satisfaction of the health care professionals with the technology and the communication experience during the therapy sessions was similar or slightly lower. In conclusion, telerehabilitation for post-knee arthroplasty is a realistic alternative for dispensing rehabilitation services for patients discharged from an acute care hospital.

Keywords: Telerehabilitation, Physical Therapy, Total Knee Arthroplasty, Videoconferencing

\section{INTRODUCTION}

Total knee arthroplasty (TKA) remains the preferred treatment for patients with incapacitating osteoarthritis. It allows these patients to regain a satisfactory quality of life. These results are reflected in the increasing number of TKA interventions performed each year in Canada (Information, 2003).

Rehabilitation is part of a successful recovery after TKA, beginning in the hospital (Bohannon \& Cooper, 1993; Dowsey, Kilgour, Santamaria, \& Choong, 1999) and continuing after discharge (NIH Consensus Development Program, 2003). It is widely recognized that home care services play a crucial role in the continuity of care after discharge from hospital for TKA. It is also recognized that home care services cannot meet the rehabilitation needs of such patients in most urban and rural areas in the province of Québec, Canada (Ministère de la Santé et des Services sociaux du Québec, 2004). In this context, alternatives to home care services must be pursued in order to meet rehabilitation needs.

Telerehabilitation, defined as the provision of remote rehabilitation services to individuals with persistent and significant disabilities via information technologies and telecommunications (Cooper et al., 2001), is growing as a complementary or alternative intervention to traditional face-to-face therapy in home care services. The rationale for telerehabilitation is to expand and facilitate the delivery of rehabilitation services to people who cannot access them due to a shortage or lack of access to services, long waiting lists for home care services, or problems getting to and from the clinic (Wakeford, Wittman, White, \& Schmeler, 2005; Winters, 2007). Clinical care that can be provided via telerehabilitation includes diagnosis, treatment and follow-up with a patient at a distance (Forducey et al., 2003). These services can be delivered in two ways: from a primary clinic to a secondary clinic, or from a clinic directly to the patient's home.

Published studies on in-home telerehabilitation for post-knee arthroplasty are limited to patient assessments using videoconferencing between two clinics (Aarnio, Lamminen, Lepisto, \& Alho, 1999; Lemaire, Necsulescu, \& Greene, 2006; Lemaire, Boudrias, \& Greene, 2001; Ohinmaa, Vuolio, Haukipuro, \& Winblad, 2002). Only one study investigated teletreatment for TKA between two clinical settings by simulating a home environment. Our 
research team conducted a proof-of-concept study where four patients received in-home telerehabilitation, but only one of these had TKA (Tousignant, Boissy, Corriveau, \& Moffet, 2006).

However, results from this proof-of-concept study showed us that providing in home telerehabilitation services can be challenging. From a telecommunication point of view, we have to rely on residential high speed Internet connections that typically have bandwidth limitations (up to $800 \mathrm{~Kb} / \mathrm{s}$ for upload streams) and are not optimized for videoconferencing applications. While these connections have the advantage of being relatively accessible, even in rural areas, their stability may be an issue when delivering one hour per day of teletreatment over a long period of time. From a clinical standpoint, if you consider that in face-to-face therapy for TKA, the physiotherapist generally provides physical assistance in mobilizing the patient's knee and in performing (or guiding patients when performing) functional exercises, questions surrounding the efficacy of teletreatment after TKA are also legitimate.

In preparation for the implementation of a randomised clinical trial on in-home telerehabilitation after TKA, we sought to explore these potential issues. The purpose of this study was to investigate in-home telerehabilitation as an alternative to face-to-face therapy for individuals at home following discharge from an acute care hospital. The objectives of this study were to: 1) assess the feasibility of providing in-home telerehabilitation services over residential high speed internet connections with $\mathrm{H} 264$ videoconferencing CODECS; 2) examine the acceptance of this service delivery method by health care practitioners and patients; and 3) obtain pilot data on its clinical efficacy.

\section{MATERIALS AND METHOD}

\section{POPULATION AND SAMPLE}

People who had TKA were recruited prior to discharge from an acute care hospital, the University Hospital of Sherbrooke (UHS). This hospital provides orthopaedic surgery for an urban (city of Sherbrooke) and rural (Eastern Townships) area in the province of Québec, Canada. The study was approved by the Sherbrooke Geriatric University Institute Ethics Committee. Potential participants were first approached by their hospital physiotherapist in the post-operative unit to determine their interest in receiving information about a research project on in-home telerehabilitation after their discharge from the hospital. Positive responses from potential participants were followed by a planned visit by a research assistant to formally explain the project. With the candidate's permission, the availability of high-speed Internet access at their home was checked by a broker with contacts from different regional Internet service providers (ISP). Once it was determined that highspeed broadband access was available, the candidate's informed consent to participate in the study was obtained. Installation of Internet access was planned with the ISP within a five-day interval. The in-home visit by the ISP technician was scheduled to coincide with the visit by a research team technician. It took about two hours per site to complete the installation and usability tests. The equipment was picked up by the research team at the end of the planned telerehabilitation period ( 2 months).

\section{TECHNOLOGICAL INFRASTRUCTURE FOR TELEREHABILITATION SERVICES}

Using results from an initial proof-of-concept study (Tousignant, Boissy, Corriveau, \& Moffet, 2006), a telerehabilitation platform consisting of two H264 videoconferencing CODECs (Tandberg 500 MXP) with integrated wide angle view cameras and remotecontrolled pan, tilt zoom (PTZ), 20-inch LCD TV and a dedicated modular software interface for user-friendly control of videoconferencing connections, PTZ camera functions and external devices, was developed (Hamel, Fontaine, \& Boissy, 2008) (Figure 1). Overviews of the telerehabilitation platform and the software interface for the clinician are illustrated in Figure 1. The platform was developed to ensure that interactions between clinicians and clients during the telerehabilitation sessions were not impeded by technology but facilitated with user-friendly interfaces. A special effort was made to provide a mousebased interface to intuitively control, from a unique screen through point-and-click or area zoom, PTZ camera functions at both sites. 


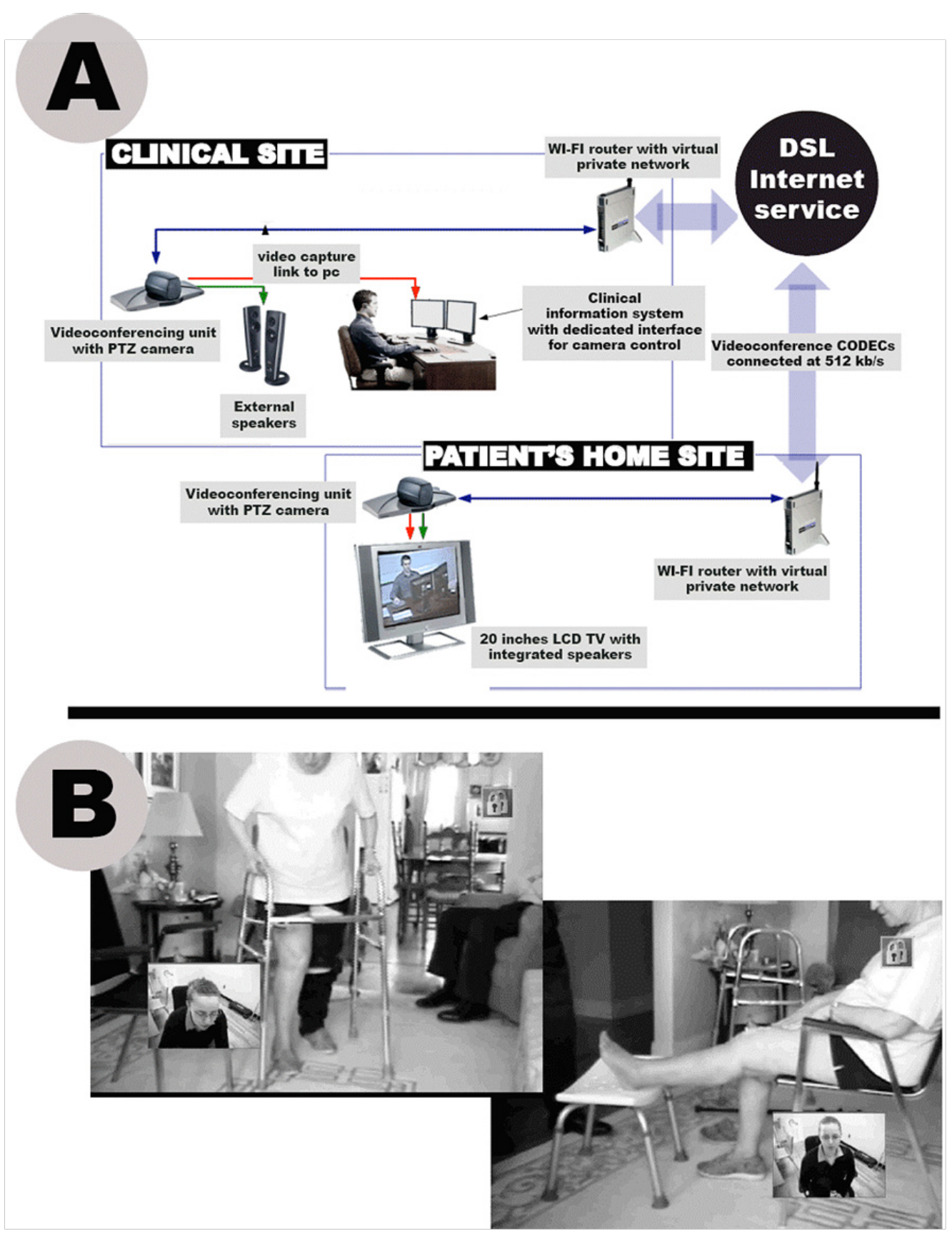

Figure 1. Technological infrastructure for telerehabilitation services

\section{OutCome MeAsures}

The major goal of rehabilitation with persons with knee arthroplasty after discharge from an acute care hospital is to recover function. To attain this goal, rehabilitation interventions must strive to reduce surgery related impairments such as limitation in knee range of motion and balance. Thus outcome measures for TKA post surgery rehabilitation are classified in two categories: 1) disability: range of motion measured by goniometry, balance measured by the Berg scale (Berg, WoodDauphinee, \& Williams, 1995; Berg, Wood-Dauphinee, Williams, \& Gayton, 1989; Berg, Wood-Dauphinee, Williams, \& Maki, 1992) and lower body strength 
measured by "30 Seconds Chair-Stand" Test (Jones, Rikli, \& Beam, 1999); and 2) function: locomotor performance in walking measured with the "Timed Up \& Go" Test (Podsiadlo \& Richardson, 1991) and functional autonomy measured with the Functional Autonomy Measurement System (SMAF) (Hebert, Carrier, \& Bilodeau, 1988).

Along with patients' satisfaction with telerehabilitation, health professional satisfaction with the use of telerehabilitation was measured with the Subjective Appreciation of Technological Quality Questionnaire, an in-house questionnaire adapted from a previous instrument (LeRouge, Garfield, \& Hevner, 2002). This questionnaire allows the clinician to assess the technical quality of each telerehabilitation session in relation to five domains: 1) reliability of the technology; 2) quality of the audio/video synchronisation; 3) quality of the image; 4) quality of the sound; and 5) quality of the operability of peripherals. Clinicians rate these aspects on a Likert scale ranging from 0 (poor quality) to 4 (high quality). Scores on these five scales are summed and expressed as a percentage. In addition, general appreciation is assessed on a scale of 0 to $100 \%$ with the following two questions: 1) Did you attain the therapeutic goals of this session? 2) What is your overall satisfaction with this session?

\section{TELETREATMENT \\ PHYSIOTHERAPY INTERVENTION}

The physiotherapy intervention was based on functional rehabilitation. A set of 10 exercises is adapted to the participant's specific condition. They are based on a functional approach to reducing impairment and improving function in daily activities. They are progressive, based on the gain obtained in each exercise for each patient. The mean duration is about 45 minutes of treatment plus 15 minutes spent on assessment and recommendations between teletreatments.

\section{Data COllection Procedures}

Design was pre/post-test without a control group (Figure 2). Once the patient returned home, an appointment was made for the assessing therapist to take the clinical measurements (T1) (disability and function). In-home telerehabilitation sessions with the participants were conducted by trained physiotherapists from the clinic, at the research centre. A family member or a friend was required to be at the participant's home during teletreatment to ensure their safety during transfers and locomotion, as well as in case of emergency. The home-based assistant received training regarding the implementation of the technology that had been installed, but not regarding the therapy. The physiotherapy program was delivered to the participants at a rate of two one-hour sessions per week for eight weeks (total of 16 sessions). Before the end of the teletreatment, the same assessing therapist visited the participant's home again to take the T2 measurements (disability, function and satisfaction). Clinical measurements were collected a third time two months post-discharge from teletreatment (T3).

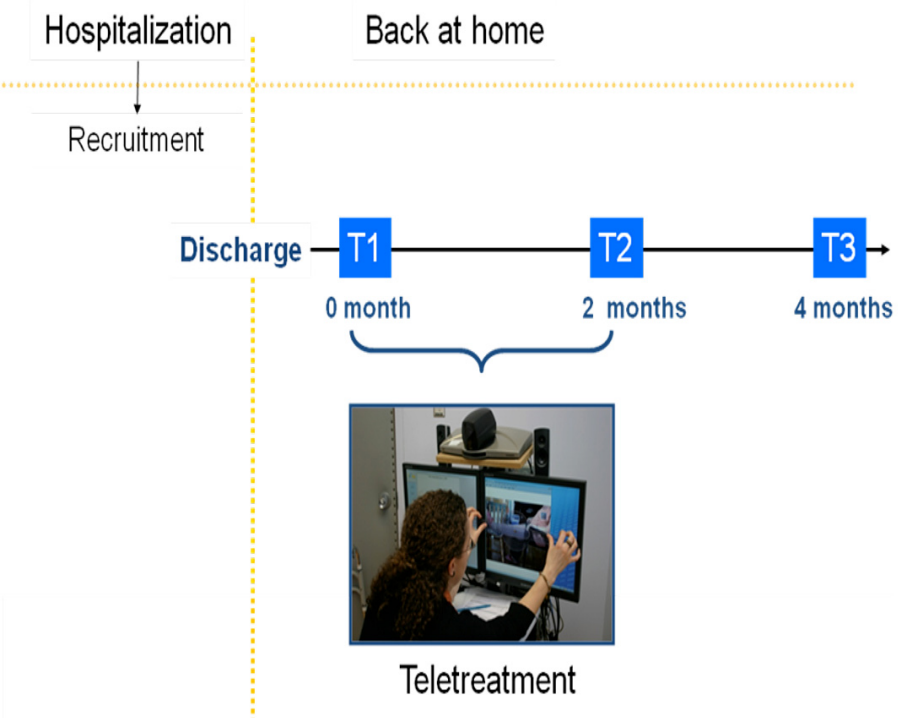

Figure 2. Design

\section{RESULTS}

Five subjects ( 3 men and 2 women) were recruited for this pilot study. Their mean age was $66.0 \pm 6.7$ years. One participant was lost during follow-up between T1 and T2, the reason being preference for face-to-face treatment despite good evolution of knee function, and their refusal to participate in any further evaluations. Descriptive statistics of the sample at the beginning of the study are given in Table 1.

\section{EFFECT OF THE TELEREHABILITATION PROGRAM}

Table 1 and Figures 3 to 7 show the effect of the telerehabilitation program on the two categories of clinical variables selected as outcome measures. Overall, teletreatment was associated with an improvement in the participants' condition over the eight weeks of therapy, as demonstrated by increased active knew range of motion (Figure 3); higher number of chair-stands in 30 seconds (Figure 4); improved balance on the Berg scale (Figure 5); increased locomotor performance in walking (Figure 6); and higher level of functional autonomy (Figure 7). This tendency to improve continued over the next two months without treatment. 


\begin{tabular}{|c|c|c|c|}
\hline & $\mathrm{T} 1$ & $\mathrm{~T} 2$ & $\mathrm{~T} 3$ \\
\hline \multicolumn{4}{|c|}{ Disability } \\
\hline \multicolumn{4}{|l|}{ Range of motion } \\
\hline Extension (degrees) & $-10.5(4.8)$ & $-5.1(6.4)$ & $-2.3(1.9)$ \\
\hline Flexion (degrees) & $85.3(8.1)$ & $105.3(4.8)$ & $112.0(3.6)$ \\
\hline Lower body strength - 30-s chair-stand test (number/30s) & $3.3(3.9)$ & $9.5(4.8)$ & $10.8(4.6)$ \\
\hline Balance - Berg (/56) & $45.5(2.4)$ & $53.5(2.1)$ & $54.0(1.8)$ \\
\hline \multicolumn{4}{|c|}{ Function } \\
\hline Locomotor performance in walking - Timed Up \& Go (s) ${ }^{1}$ & $26.7(5.4)$ & $11.5(3.1)$ & $10.7(2.9)$ \\
\hline Functional autonomy - SMAF $(/ 87)^{1}$ & $19.9(2.7)$ & $5.8(4.1)$ & $3.9(2.3)$ \\
\hline
\end{tabular}

Table 1 Descriptive Statistics for Clinical Outcome Measures of Telerehabilitation Program

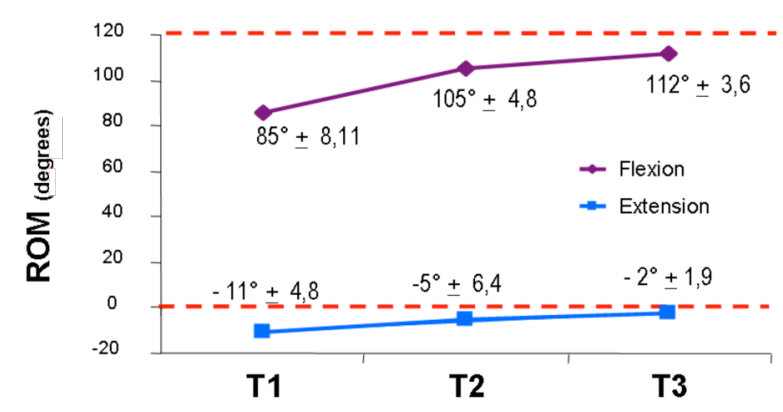

Figure 3. Active Knee Range of Motion

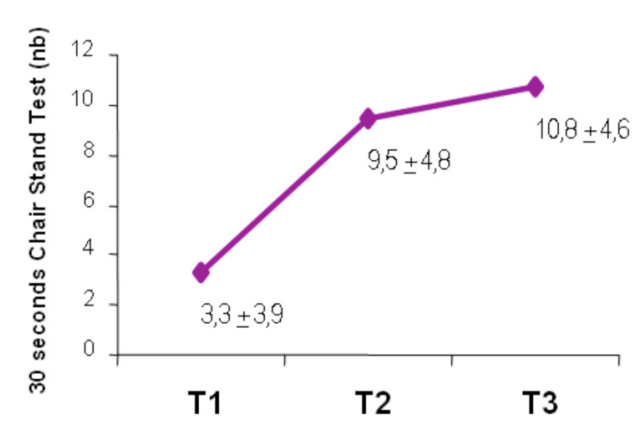

Figure 4. 30 Seconds Chair Stand Test

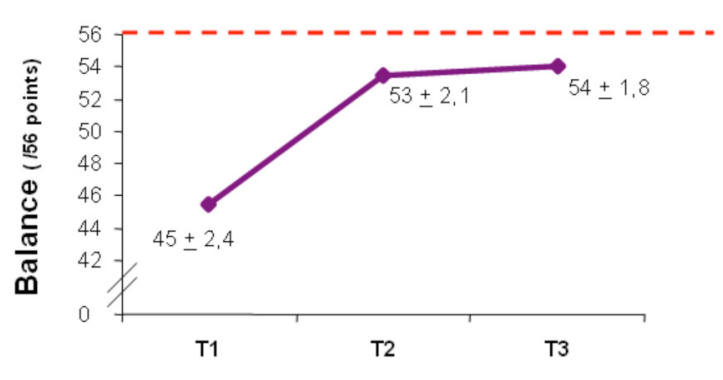

Figure 5. Berg Test Score

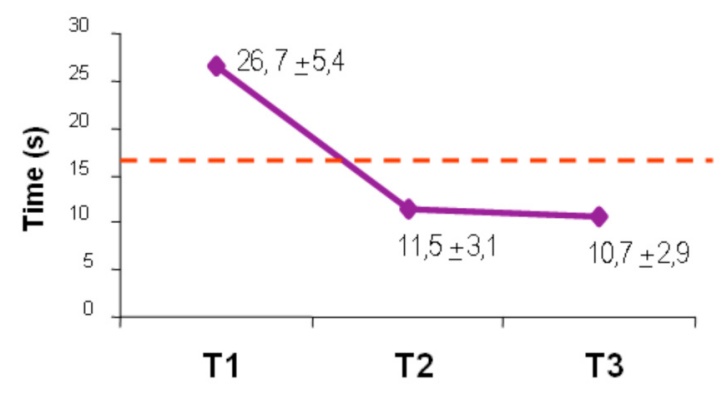

Figure 6. Timed Up and Go Test (TUG) 


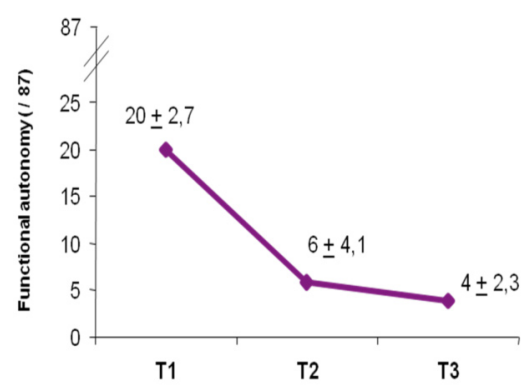

Figure 7. Functional Autonomy Measurement System (SMAF)

\section{SATISFACTION WITH PHYSIOTHERAPY TELETREATMENT}

The satisfaction of the participants with teletreatment was very high: $94.7 \pm 7.8 \%$ (maximal score being 100\%). None of the components of satisfaction were less than satisfactory: clinician $(96.6 \pm 4.2 \%)$, organization of services (92.4 $\pm 10.2 \%)$, and delivery of services (95.2 \pm 9.6\%).

Subjective satisfaction of the clinician with the technical performance of the platform was also high: $83.4 \pm 2.0 \%$. Satisfaction with the clinical use of the platform was similar or slightly lower: patient-therapist relationship (84.4 $\pm 13.8 \%$ ); therapeutic goal attained (78.4 $\pm 18.9 \%)$, and overall satisfaction $(76.6 \pm 14.1 \%)$.

\section{DISCUSSION}

The objective of this pilot study was to investigate the feasibility of delivering physiotherapy teletreatment using the Internet between a patient's home and a clinical setting for patients after knee arthroplasty. To our knowledge, this is the first study that illustrates the feasibility of carrying out a complete rehabilitation program for follow-up of post-knee arthroplasty, including specific therapeutic exercises and evaluations done remotely at patients' homes under real conditions. While the very small number of subjects involved in this study makes it difficult to draw clear conclusions, we may highlight some trends: 1) physiotherapy teletreatment delivered over a long period of time is a feasible contingent; 2) physiotherapy teletreatment seems to be effective in reducing disability (range of motion, balance and muscle strength) and improving function (walking and functional autonomy); and 3) health care professionals, as well as patients, appear satisfied with this service delivery method without a face-to-face session.

Our pilot study shows the clinical relevance of in-home teletreatment for this type of patient. The challenge was to ensure that the physiotherapist can interact with the patient, provide specific instructions and feedback regarding satisfaction, and maintain a good patienttherapist relationship without the face-to-face advantage. Given that telerehabilitation is a compromise compared to face-to-face intervention, we may argue that this compromise is acceptable and has the potential to increase access to services in urban and rural areas with high speed Internet services in two ways: 1) more patients can be treated each day by telerehabilitation compared to traditional home care services, where the therapist needs to travel to the patient's home; and 2) some rural areas cannot be served by home care services.

A key focus of our study was to demonstrate that residential high-speed Internet connections are stable enough to deliver the video and sound quality required in physiotherapy interventions in home telerehabilitation. Indeed, video quality is more important in our context than in other telehealth uses such as teleconsultation in psychiatry namely because a high definition was required in addition to the need to quickly move the camera in different planes and change the focus. Our study suggests that the quality of the communication experience provided under this context is good enough to meet the clinician's expectations. However, it should be noted that with state of the art videoconferencing equipments such as those used in this study; image pixelisation, video frame loss and sometime disconnections were reported but did not affect the overall clinical experience.

Our study shows that telerehabilitation treatment seems to be effective, as evidenced by improvements in physical function across the board. We cannot, however, attribute the improvement solely to the telerehabilitation treatment since natural recovery and other variables were not controlled for in this design without a control group. Knowing that teletreatment is feasible and acceptable to clinicians and patients, the next step will be to conduct controlled trials in order to compare the cost-benefit of two treatment alternatives, such as telerehabilitation and home visits.

\section{CONCLUSION}

Telerehabilitation seems to be a practical alternative to home visits by a physiotherapist for dispensing rehabilitation services. This new delivery method appears acceptable to patients and health professionals, although both must modify their expectations. A residential Internet network provides enough quality to make in-home teletreatment feasible, even though disconnections can reduce user satisfaction. Future research is needed with a randomized controlled trial to measure the efficacy of this novel way of delivering rehabilitation services. 


\section{ACKNOWLEDGEMENTS}

This research was supported in part by a grant received from the Research Centre on Aging. We would like to thank the participants on this project. Correspondence concerning this article should be addressed to Michel Tousignant, Research Centre on Aging, Sherbrooke Geriatric University Institute, 1036 Belvédère Sud, Sherbrooke, Québec J1H 4C4 Canada. E-mail: michel. tousignant@usherbooke.ca

\section{REFERENCES}

Aarnio, P., Lamminen, H., Lepisto, J., \& Alho, A. (1999). A prospective study of teleconferencing for orthopaedic consultations. Journal of Telemedicine and Telecare, 5(1), 62-66.

Berg, K., Wood-Dauphinee, S., \& Williams, J. I. (1995). The Balance Scale: Reliability assessment with elderly residents and patients with an acute stroke. Scand J Rehabil Med, 27(1), 27-36.

Berg, K. O., Wood-Dauphinee, S., Williams, J. L., \& Gayton, D. (1989). Measuring balance in the elderly: Preliminary development of an instrument. Physiotherapy Canada, 41, 304-311.

Berg, K. O., Wood-Dauphinee, S. L., Williams, J. I., \& Maki, B. (1992). Measuring balance in the elderly: Validation of an instrument. Canadian Journal of Public Health, 83 Suppl 2, S7-11.

Bohannon, R. W., \& Cooper, J. (1993). Total knee arthroplasty: Evaluation of an acute care rehabilitation program. American Academy of Physical Medicine and Rehabilitation, 74(10), 1091-1094.

Cooper, R. A., Fitzgerald, S. G., Boninger, M. L., Brienza, D. M., Shapcott, N., Cooper, R., et al. (2001). Telerehabilitation: Expanding access to rehabilitation expertise. Proceedings of the IEEE, 89(8), 1174-1193.

Dowsey MM, Kilgour ML, Santamaria NM, \& Choong, PF. (1999). Clinical pathways in hip and knee arthroplasty: A prospective randomised controlled study. Medial Journal Australian, 170(2), 59-62.

Forducey, P. G., Ruwe, W. D., Dawson, S. J., ScheidemanMiller, C., McDonald, N. B., \& Hantla, M. R. (2003). Using telerehabilitation to promote TBI recovery and transfer of knowledge. NeuroRehabilitation, 18(2), 103111.

Hamel, M., Fontaine, R., \& Boissy, P. (2008). In-home telerehabilitation for geriatric patients. IEEE Engineering in Medicine and Biology Magazine, 27(4), 29-37.

Hebert, R., Carrier, R., \& Bilodeau, A. (1988). The Functional Autonomy Measurement System (SMAF): Description and validation of an instrument for the measurement of handicaps. Age Ageing, 17(5), 293-302.

Information, C. I. f. H. (2003). Number of total hip and knee replacement procedures and percent change in Canada 1995-2001: http://secure.cihi.ca.

Jones, C. J., Rikli, R. E., \& Beam, W. C. (1999). A 30-s chair-stand test as a measure of lower body strength in community-residing older adults. Research Quarterly for Exercise and Sport, 70(2), 113-119.

Lemaire, E., Necsulescu, L., \& Greene, G. (2006). Service delivery trends for a physical rehabilitation outreach program. Disability and Rehabilitation, 28(21), 13491359.

Lemaire, E. D., Boudrias, Y., \& Greene, G. (2001). Low-bandwidth, Internet-based videoconferencing for physical rehabilitation consultations. Journal of Telemedicine and Telecare, 7(2), 82-89.

LeRouge, C., Garfield, M. J., \& Hevner, A. R. (2002). Quality attributes in telemedicine video conferencing. Paper presented at the 35th Annual Hawaii International Conference on Systems Sciences, Hawaii. 
Ministère de la Santé et des Services sociaux du Québec. (2004). Chez soi : Le premier choix. Précisions pour favoriser l'implantation de la politique de soutien à domicile. Québec: La Direction des communications, MSSS-Québec.

NIH Consensus Development Program. (2003). NIH Consensus Development Conference on Total Knee Replacement. Final Statement.

Ohinmaa, A., Vuolio, S., Haukipuro, K., \& Winblad, I. (2002). A cost-minimization analysis of orthopaedic consultations using videoconferencing in comparison with conventional consulting. Journal of Telemedicine and Telecare, 8(5), 283-289.

Podsiadlo, D., \& Richardson, S. (1991). The timed "Up \& Go": A test of basic functional mobility for frail elderly persons. Journal of the American Geriatrics Society, 39(2), 142-148.

Tousignant, M., Boissy, P., Corriveau, H., \& Moffet, H. (2006). In home telerehabilitation for older adults after discharge from an acute hospital or rehabilitation unit: $A$ proof-of-concept study and costs estimation. Disability and Rehabilitation Assistive Technology, 1(4), 209-216.

Wakeford, L., Wittman, P. P., White, M. W., \& Schmeler, M. R. (2005). Telerehabilitation position paper. Am J Occup Ther, 59(6), 656-660.

Winters, J. M. (2007). Videoconferencing and telehealth technologies can provide a reliable approach to remote assessment and teaching without compromising quality. J Cardiovasc Nurs, 22(1), 51-57. 